\title{
Optimal Management of Hereditary Angioedema: Shared Decision-Making
}

This article was published in the following Dove Press journal:

Journal of Asthma and Allergy

\begin{abstract}
Aleena Banerji ${ }^{1}$
John Anderson ${ }^{2}$

Douglas T Johnston ${ }^{3}$

'Division of Rheumatology, Allergy and Immunology, Massachusetts General Hospital, Boston, MA, USA; ${ }^{2}$ Clinical Research Center of Alabama, Birmingham, AL, USA; ${ }^{3}$ Asthma and Allergy Specialists, Charlotte, NC, USA
\end{abstract}

Video Abstract

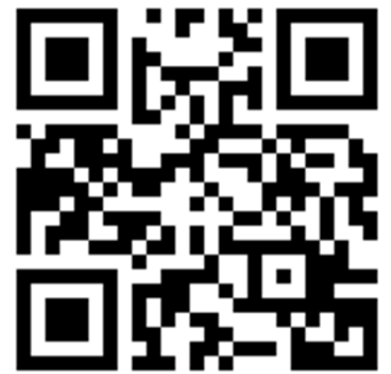

Point your SmartPhone at the code above. If you have a QR code reader the video abstract will appear. Or use: https://youtu.be/wwl_GBviKOU
Correspondence: Aleena Banerji

6 Aaron Road, Lexington, MA, 0242I,

USA

Tel $+|-6| 7-308-5975$

Fax + I-617-724-0239

Email ABANERJI@mgh.harvard.edu

\begin{abstract}
All patients with hereditary angioedema (HAE) must have access to on-demand therapy to treat attacks and may benefit from prophylactic therapy to reduce the attack frequency. Treatment decisions should be individualized, based on patient preferences and needs. One method for facilitating individualized therapy is shared decision-making (SDM), a widely used methodology for making treatment decisions among multiple therapeutic options. We propose a three-phase "3D" model (Discover, Discuss, Decide) for SDM in HAE. The Discover phase focuses on improving the physician's understanding of the patient's needs and understanding of the available therapeutic choices. The Discuss phase considers the alternatives, allowing a collaborative, informed treatment selection in the Decision phase. The 3D model is an ongoing, iterative process based on the patient's changing needs and response to therapy. Uncovering the patient's therapy goals through appropriate questions during these phases can help uncover relevant information for treatment selection information. SDM based on the $3 \mathrm{D}$ model can be a beneficial tool for optimizing therapy in HAE.
\end{abstract}

Keywords: prophylaxis, hereditary angioedema, individualized therapy, patient preference, on-demand therapy, adherence, quality of life, shared decision-making

\section{Introduction}

A generation ago, physicians caring for patients with hereditary angioedema (HAE) focused on preventing mortality. A decade ago, as novel therapies became available in the United States, the focus shifted to keeping the patient out of the hospital. Now, with multiple effective preventive and on-demand therapies available, the focus has shifted further, to designing individualized preventive strategies that aim to optimize the patient's quality of life (QoL). Shared decision-making (SDM) is a key component of creating an individualized treatment plan. Treatment decisions should be built on mutual understanding of the patient's needs and goals as well as the potential benefits and harms of the treatment options. This article outlines an approach to facilitate effective, efficient SDM in real-world HAE practice.

HAE is a rare but potentially disabling and life-threatening genetic disorder that manifests as recurrent, unpredictable attacks of localized bradykinin-mediated subcutaneous or mucosal edema. It is an inherited autosomal dominant disorder caused by mutations in the SERPING1 gene, which lead to deficiency of functional $\mathrm{C} 1$ esterase inhibitor (C1-INH). ${ }^{1}$ The two most common forms are HAE with $\mathrm{C} 1-\mathrm{INH}$ deficiency (C1-INH-HAE), which may be Type 1 (approximately $85 \%$ of cases) or Type 2 (approximately $15 \%$ of cases). Rare other types with normal C1-INH levels

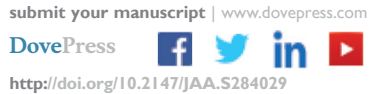


and function (nlC1-INH-HAE) have also been identified. ${ }^{2,3}$ The prevalence is estimated at approximately 1 in 50,000 patients (range 1 in 10,000 to 1 in 100,000). ${ }^{1,4}$

Attacks usually affect the skin of the extremities, face, trunk, or genitalia, or the submucosa of the intestines or upper respiratory tract, with a typical duration of 2 to 5 days per attack if untreated. ${ }^{1,2}$ Cutaneous attacks affect almost all patients with HAE, causing non-pitting, nonpruritic edema with no urticaria or wheals. In some cases, prodromal erythema marginatum may precede $\mathrm{HAE}$ attacks. Erythema marginatum may resemble urticaria, causing diagnostic difficulties. ${ }^{3}$ The swelling can be temporarily disabling or disfiguring. ${ }^{1,2}$ Abdominal attacks are common, affecting $70 \%$ to $90 \%$ of patients with HAE. ${ }^{2,5}$ Manifestations range from mild, intermittent abdominal discomfort to bowel obstruction with excruciating pain, vomiting, and/or diarrhea. ${ }^{1,2}$ Laryngeal attacks are uncommon, making up $\sim 1 \%$ of all attacks, but may cause lifethreatening asphyxiation. ${ }^{1}$

Patients with HAE face a heavy burden of disease. Attacks impair the patient's ability to perform activities of daily living, decrease productivity at work or in school, and inhibit social activities. ${ }^{6}$ Numerous studies have confirmed impairment in multiple dimensions of healthrelated quality of life (HRQoL) in patients with HAE. ${ }^{6-12}$ Anxiety and depression related to the ever-present risk of potentially severe or life-threatening attacks are common. ${ }^{6,10}$ Further, treatment for HAE also imposes an additional burden: Most prophylactic and all on-demand therapies are administered by subcutaneous (SQ) or intravenous (IV) injection, which can be time-consuming and may cause adverse reactions.

Treatment of HAE uses two broad strategies for medical management: On-demand therapy, to minimize the impact of HAE attacks, and prophylactic therapy in appropriate patients, to reduce the frequency and severity of attacks. Because attacks are unpredictable and potentially life-threatening, readily available, effective, on-demand therapy is essential for all patients. ${ }^{3,13,14}$ Individualized long-term prophylactic therapy should be considered for all patients based on factors such as disease activity, attack frequency, patient QoL, ability to control attacks with ondemand therapy, and patient preference. ${ }^{3,14,15}$ Patients should also receive short-term prophylaxis in advance of planned dental and surgical procedures to reduce the risk that the procedure will trigger an attack. ${ }^{3,14,15}$

The available therapies differ considerably in their route and frequency of administration, convenience, tolerability and adverse effects, and costs. Patients may also have preferences arising from their family's experience, lifestyle, knowledge, and opinions about HAE. Box 1 presents common concerns that arise in HAE. Optimizing treatment by individualizing therapy plans is a key challenge in caring for patients with HAE. SDM addresses this challenge by explicitly bringing the patient in as a partner in a collaborative decisionmaking process. This article presents suggestions and a model for using SDM in the real-world management of patients with HAE, as well as three typical but fictional case studies designed to illustrate how SDM can be applied in real-world clinical practice.

Box I Common Examples of Concerns Related to HAE Treatment Decision-Making

Medical History

- Attack history (frequency, location, severity, impact on QoL and well-being)

- Patient experience with on-demand therapy (response, achievement of control, ability to administer, adverse effects)

- Patient experience with and response to long-term prophylaxis (efficacy, administration, adverse effects)

- History of adverse effects of treatment

- Comorbidities (eg, high thromboembolic risk)

Patient Goals

- Desire to live a "normal" life/minimize impact of attacks on QoL

- Desire to engage in specific activities (eg, sports) with less fear of an HAE attack

- HAE-related limitations for work, school, and/or social functions

- Travel

- Family planning

- Patient's support system

Patient Preference

- Anxiety or fear of potential attacks

- Preferences for route of administration; ability and willingness to self-administer (eg, burden of treatment, needle phobia)

- Family or personal experience with and perceptions of HAE and specific HAE medications

- Minimize impact of disease on day-to-day living

- Minimize treatment burden

- Concerns about adverse effects

- Availability of caregiver support

- Cost and access/medical insurance

\section{Access}

- Cost of treatment/insurance coverage

- Distance from clinic

- Distance/time to the nearest emergency department

Abbreviations: HAE, hereditary angioedema; QoL, quality of life. 


\section{Fictional Case Presentation}

A 35-year-old male with Type 1 HAE has experienced attacks every 2-3 months since being diagnosed at 19 years of age. He manages the attacks with selfadministered, on-demand IV complement 1 esterase inhibitor (CI-INH).

In the past few years, deteriorating venous access has caused increasing difficulty with self-administration. In his current job, self-administration is awkward, so he often delays treatment and does not treat all attacks. His attacks have become more severe and prolonged, including two painful abdominal attacks that required an ED visit. The patient has also developed increasing anxiety and fear about his attacks, causing substantial strain on his job, social life, and lifestyle. He was recently promoted to a position that requires frequent travel, and he is extremely anxious about a possible HAE attack while traveling.

After the second abdominal attack, the patient asked his physician about changing therapy. The physician and patient used SDM to discuss the available on-demand and prophylactic therapies and decided to try an on-demand therapy that can be self-administered by SC injection. At a follow-up visit 3 months later, the patient reported one attack, which was mild and resolved rapidly after ondemand treatment. However, prodromal symptoms appeared during an important business meeting and were embarrassing. Based on this experience, the patient and physician agreed to a 6-month trial of long-term prophylaxis along with continued use of an on-demand therapy that could be self-administered by SC injection and carried during travel.

\section{Overview of Shared Decision-Making}

Shared decision-making has been defined as

an approach where clinicians and patients share the best available evidence when faced with the task of making decisions, and where patients are supported to consider options, to achieve informed preferences. ${ }^{16}$

SDM is especially appropriate in therapeutic areas where there are multiple choices of therapy and patient preference is important, such as oncology, asthma, and palliative care. ${ }^{17}$ SDM is based on two primary ideas: The ethical imperative that patients should be involved in determining their own care, and the need to make decisions based on accurate information. A wealth of data indicates that SDM improves care when used appropriately. ${ }^{16-19}$ Studies on the impact of SDM on care have found that SDM can empower and educate patients, ${ }^{18,20}$ improve patient adherence and satisfaction, ${ }^{18,21,22}$ facilitate decision-making, ${ }^{18}$ decrease costs, ${ }^{23,24}$ and improve outcomes. ${ }^{21,25}$ The principles of care for patients with HAE, particularly the need for individualized therapy, align with the principles of SDM. Thus, the established principles of SDM can provide a useful model for individualized decision-making in HAE.

\section{Discover, Discuss, Decide:}

\section{A proposed “3D” Model for Shared Decision-Making in Real-World HAE Practice}

We propose a three-stage model adapted for HAE from a more general model developed by Elwyn et al (Figure 1). ${ }^{16,26}$ The three stages are Discover, Discuss, and Decide, abbreviated as 3D. In the Discover phase, the physician works collaboratively with the patient to discover and understand the patient's needs and preferences, explains the reasonable therapeutic choices that are available, and offers decision support. The Discuss phase focuses on a discussion of reasonable alternatives aligned to the patient's needs and preferences. Finally, in the Decide phase, the physician and patient collaboratively make a treatment decision that is informed by an improved mutual understanding of the alternative therapies and the patient's needs and preferences. Shared decision-making is an ongoing, iterative process in which treatment decisions are updated based on the patient's changing needs and response to therapy.

\section{Fictional Case Presentation}

A 25-year-old male with Type 1 HAE was diagnosed 4 years ago and uses androgens for long-term prophylaxis. The patient's father also has HAE and uses the same regimen. Although the patient was informed of the risks associated with androgens, he was satisfied with androgen therapy until he was recently diagnosed with hypertension. The patient's primary care physician strongly advised that he stop androgen therapy to reduce his cardiovascular risk, and the allergist strongly supported the recommendation.

The allergist initiated a conversation using SDM. In the Discover phase, the allergist clarified that several options were available for prophylaxis beyond androgens, offered support in decision-making, and asked the patient about his 


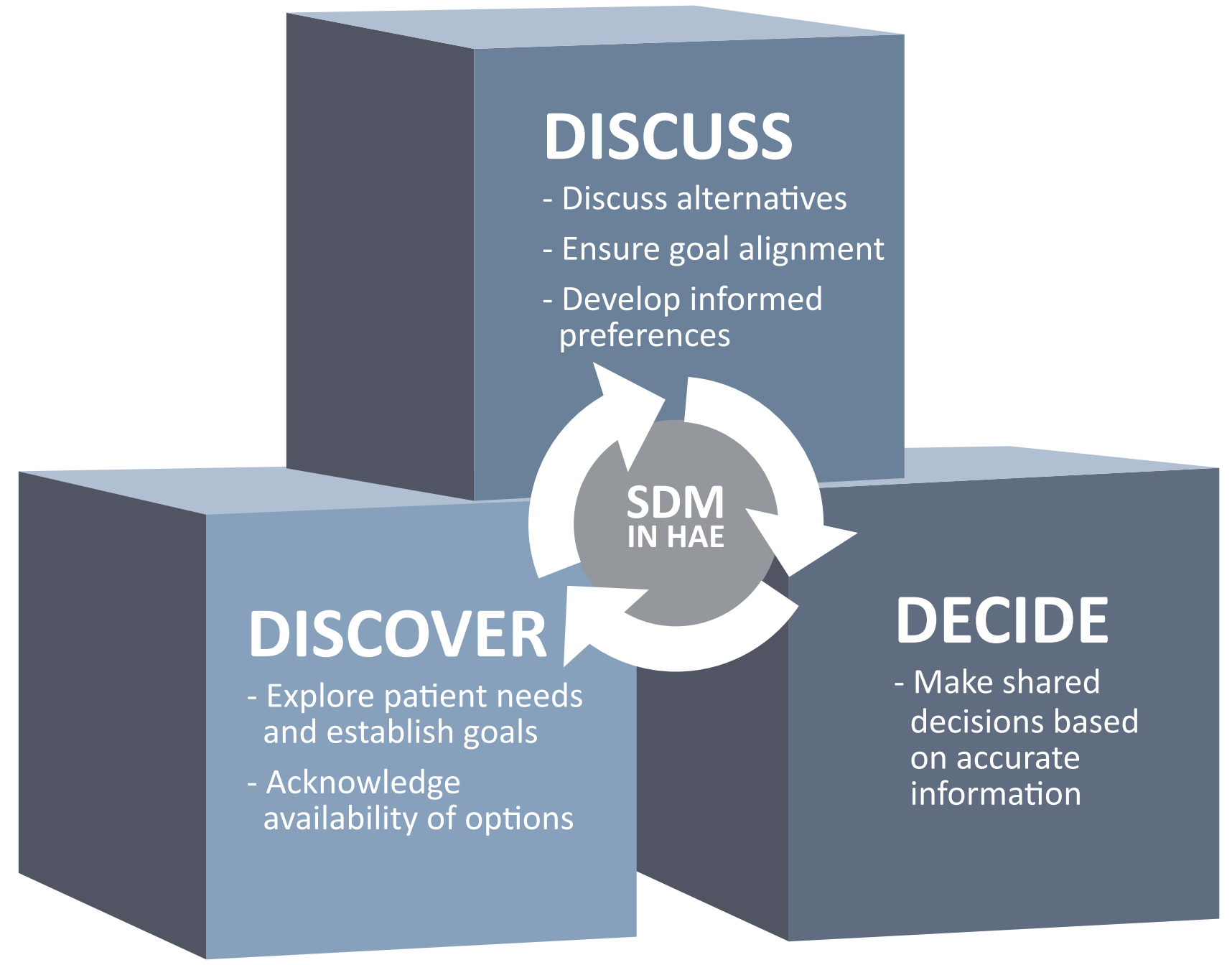

Figure I Proposed 3D model (Discover, Discuss, Decide) for SDM in HAE. Abbreviations: HAE, hereditary angioedema; SDM, shared decision-making.

goals of therapy. It was clear that the patient followed his father's example without considering the available alternatives. The physician provided the patient with information on HAE, recommended the Hereditary Angioedema Association (HAEA) website, and set up a follow-up visit. The Discuss and Decide phases both occurred during the follow-up visit. The patient did not want to continue taking androgens but was concerned about repeated injections. The allergist reviewed several prophylactic treatment options, and the patient and physician mutually decided to switch to an alternative long-term prophylactic treatment and follow up in 3 months to discuss the results with the new therapy. This process could have produced different outcomes, depending on the patient's situation and needs, such as decreasing the dose of androgen therapy or discontinuing prophylactic treatment.
The Discover phase often begins with a general check on the patient's HAE status, covering topics such as the frequency and severity of attacks, their current HAE medications, and how well they control the disease. After this, broad, general questions can be useful to open an SDM discussion. What are the patient's goals? What is the patient's status in relation to those goals? How can the patient achieve their goals? For many patients, the goal is "having a normal life." This requires a discussion to understand what "having a normal life" means for the patient. Gaining insight into the patient's goals, situation, and perception of HAE is critical to collaboratively identifying an appropriate therapy. Several specific issues should be considered in the Discover or Discussion phases, including the patient's lifestyle, the impact of HAE, the patient's personal experience and preferences, and the patient's medical history (Box 1). 
As part of the Discover phase, the physician and patient evaluate whether to consider a change in therapy. If the patient and physician are both satisfied with current therapy, no change is needed, and the SDM discussion can continue at the next visit. If a change is needed, the physician should introduce the available therapeutic choices that align with the patient's needs and preferences. Patients should be provided with patient-level information on HAE and HAE treatments. Patients may also benefit from reliable online information sources such as the HAEA website. It may also be possible to introduce patients to an expert in the field of HAE via a virtual visit. Patients may need time to learn about the available treatment choices. Therefore, it is useful to spread out the SDM process by completing the Discussion and/or Decision phases in later visits.

\section{Fictional Case Presentation}

A 22-year-old female with Type $1 \mathrm{HAE}$ was diagnosed at 12 years of age. Since then, she has had an average of 1-2 attacks every month. The attacks have been bothersome but do not keep her from work or other important activities when treated with IV C1-INH on-demand therapy. She recently finished college, found a job, and lives away from her family for the first time. Self-treatment is more difficult now that she lives independently, particularly because she is needle-phobic. In addition, managing an attack is awkward at work and during her social functions. She tried androgens for prophylaxis when she started college but discontinued them due to side effects. She has also tried each available on-demand medication and found that IV C1-INH is the most effective for her.

During the Discover phase of SDM, the patient's allergist asked about family planning and learned that the patient is engaged to be married and plans to have children. During the Discussion and Decision phases, the patient and allergist agreed that prophylaxis was needed. Based on the patient's goals, they agreed that androgens should be avoided and elected to start a trial of a non-IV prophylactic agent such as subcutaneous $\mathrm{C} 1-\mathrm{INH}$.

\section{The Discussion Phase}

The Discussion phase typically focuses on one or more of three key decisions: Options for on-demand therapy, whether to use prophylactic therapy, and options for prophylactic therapy. This phase is also a good opportunity to discuss developing or updating the patient's HAE action plan. Ondemand only versus on-demand and prophylactic treatment
Box 2 Factors to Consider in Deciding Whether to Use Prophylaxis. Note: Data from Maurer et $\mathrm{al}^{3}{ }^{3}$ Zuraw et $\mathrm{al},{ }^{13}$ and Betschel et al. ${ }^{14}$

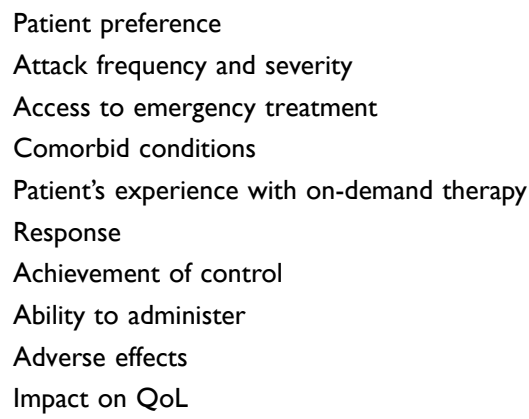

is an important conversation within the Discussion phase. Patient-related concerns discussed in Box 2 should also be considered during the discussion. When discussing the various options, acknowledging that there are several viable alternatives is important. However, further discussion should usually focus on the options that are most appropriate for the patient, based on the patient's needs and preferences.

Finally, in the Decision phase, the physician and patient collaboratively make an informed therapeutic decision based on the knowledge and understanding developed in the previous phases, which have usually identified a few treatment choices. In some cases, the patient comes in with a firm pre-existing preference, often based on family history or the patient's research. In this situation, it is important to ensure that the patient understands the benefits and risks of the various options, and for the physician to respect reasonable decisions by the patient's reasonable decisions, even if they differ from the physician's recommendations. Some patients may be hesitant because they are afraid of making a "wrong" decision. Offering the selected treatment as a trial for a limited duration, explaining that SDM is an ongoing process and that a different therapy can be selected if needed, and reassurance that the physician will not support an unreasonable decision may help address the hesitation.

There are several barriers to the effective use of SDM in real-world practice. Possibly the most important is that SDM takes more time than a conventional office visit. Physicians may need to allocate more time for visits that involve SDM for HAE, spread the decision-making process over several visits, or provide patients with information on HAE and ask them to read it before the SDM discussion. A nurse, rather than the physician, can manage patient education and many administrative tasks associated with SDM. Telemedicine can 
also be a useful alternative. Co-management with a physician who has expertise in HAE may help physicians who are not comfortable with SDM.

Disconnects between the patients' and the physicians' beliefs and priorities often present obstacles. Patients may have limited knowledge of HAE and need education in the disease state and treatment. Providing accurate, unbiased, patient-level information may be challenging. Although reliable sources such as the HAEA are available online, they may provide too much information for some patients. Patients may have preconceived biases, often based on other family members' experiences, which can be quite difficult to change. In these situations, patient education with an accurate, fair presentation of the treatment options is essential, as is respect for the patient's preferences, even when they differ from the physician's. A few patients prefer to avoid SDM and want the physician to decide for them. Often, physicians can bring these patients into the discussion by explaining that they can make better decisions if they understand the patient's needs and goals.

Administrative and payer-related issues such as requirements for prior authorization and difficulty obtaining approval often present barriers to using therapies selected by an SDM process. Cost and access may also be an issue for patients. The patient assistance programs sponsored by many manufacturers of HAE medications may help overcome these barriers.

\section{Conclusion}

HAE is a rare, potentially fatal condition with a very high burden of disease. Patients' expectations vary and may be based on family experiences from an era when treatment options were limited. Current therapies give patients a chance for a brighter future with more treatment options and an improved ability to tailor therapy to their needs and preferences. SDM, based on the 3D model (Discover, Discuss, Decide), can help patients achieve this brighter future by providing up-to-date, accurate information on treatment options, helping physicians understand patient goals, and ultimately optimizing therapies to improve QoL.

\section{Acknowledgments}

Editorial assistance was provided by Medical Leverage with funding from Biocryst Pharmaceuticals, Inc. Medical writing support was provided by Edward K. Baldwin, PhD.

\section{Author Contributions}

All authors made a significant contribution to the work reported, whether that is in the conception, study design, execution, acquisition of data, analysis and interpretation, or in all these areas; took part in drafting, revising or critically reviewing the article; gave final approval of the version to be published; have agreed on the journal to which the article has been submitted; and agree to be accountable for all aspects of the work.

\section{Disclosure}

Aleena Banerji has received research grants from Takeda and Biocryst, is a consultant for Takeda, Biocryst, CSL Behring, Pharming, Pharvaris, and Kalvista, and reports personal fees from Takeda, Biocryst, CSL, Pharming, Pharvaris, and Kalvista, outside the submitted work. John Anderson is a speaker and consultant for CSL Behring, Takeda, and Pharming, and a consultant for Biocryst, has served as a principal investigator on trials sponsored by CSL Behring, Takeda, Pharming, and Biocryst, and reports personal fees, clinical research and advisory board from/for Biocryst, clinical research, speaker bureau and advisory boards for CSL Behring, Takeda, and Pharming, outside the submitted work. Douglas Johnston is on the speaker's bureau for CSL Behring, Pharming, Shire/Takeda, and Pfizer and is a consultant for Aimmune, Allakos, Biocryst, CSL Behring, Pharming, Shire/Takeda, and Regenxbio, and reports personal fees from Biocryst, Takeda, RegenXbio, Pharming, CSL Behring, Pfizer, Aimmune, and Kalvista, outside the submitted work. The authors report no other potential conflicts of interest for this work.

\section{References}

1. Longhurst HJ, Bork K. Hereditary angioedema: an update on causes, manifestations and treatment. Br J Hosp Med (Lond). 2019;80 (7):391-398. doi:10.12968/hmed.2019.80.7.391

2. Lumry WR. Overview of epidemiology, pathophysiology, and disease progression in hereditary angioedema. Am J Manag Care. 2013;19(7 Suppl):s103-110.

3. Maurer M, Magerl M, Ansotegui I, et al. The international WAO/ EAACI guideline for the management of hereditary angioedema the 2017 revision and update. Allergy. 2018;73(8):1575-1596. doi:10.1111/all.13384

4. Cicardi M, Aberer W, Banerji A, et al. Classification, diagnosis, and approach to treatment for angioedema: consensus report from the Hereditary Angioedema International Working Group. Allergy. 2014;69(5):602-616. doi:10.1111/all.12380

5. Bork K, Meng G, Staubach P, Hardt J. Hereditary angioedema: new findings concerning symptoms, affected organs, and course. Am J Med. 2006;119(3):267-274. doi:10.1016/j.amjmed.2005.09.064 
6. Banerji A, Davis KH, Brown TM, et al. Patient-reported burden of hereditary angioedema: findings from a patient survey in the United States. Ann Allergy Asthma Immunol. 2020;124(6):600-607. doi:10. 1016/j.anai.2020.02.018

7. Caballero T, Aygören-Pürsün E, Bygum A, et al. The humanistic burden of hereditary angioedema: results from the Burden of Illness Study in Europe. Allergy Asthma Proc. 2014;35(1):47-53. doi:10.25 00/aap.2013.34.3685

8. Longhurst H, Bygum A. The humanistic, societal, and pharmaco-economic burden of angioedema. Clin Rev Allergy Immunol. 2016;51(2):230-239. doi:10.1007/s12016-016-8575-2

9. Caballero T, Prior N. Burden of illness and quality-of-life measures in angioedema conditions. Immunol Allergy Clin North Am. 2017;37 (3):597-616. doi:10.1016/j.iac.2017.04.005

10. Fouche AS, Saunders EF, Craig T. Depression and anxiety in patients with hereditary angioedema. Ann Allergy Asthma Immunol. 2014;112 (4):371-375. doi:10.1016/j.anai.2013.05.028

11. Lumry WR, Castaldo AJ, Vernon MK, Blaustein MB, Wilson DA, Horn PT. The humanistic burden of hereditary angioedema: impact on health-related quality of life, productivity, and depression. Allergy Asthma Proc. 2010;31(5):407-414. doi:10.2500/aap.2010.31.3394

12. Nordenfelt P, Dawson S, Wahlgren CF, Lindfors A, Mallbris L, Björkander J. Quantifying the burden of disease and perceived health state in patients with hereditary angioedema in Sweden. Allergy Asthma Proc. 2014;35(2):185-190. doi:10.2500/aap.2014.35.3738

13. Zuraw BL, Banerji A, Bernstein JA, et al. US Hereditary Angioedema Association Medical Advisory Board 2013 recommendations for the management of hereditary angioedema due to $\mathrm{C} 1$ inhibitor deficiency. $J$ Allergy Clin Immunol Pract. 2013;1 (5):458-467. doi:10.1016/j.jaip.2013.07.002

14. Betschel S, Badiou J, Binkley K, et al. The International/Canadian Hereditary Angioedema Guideline. Allergy Asthma Clin Immunol. 2019;15:72. doi:10.1186/s13223-019-0376-8

15. Banerji A, Busse P, Christiansen SC, et al. Current state of hereditary angioedema management: a patient survey. Allergy Asthma Proc. 2015;36(3):213-217. doi:10.2500/aap.2015.36.3824

16. Elwyn G, Frosch D, Thomson R, et al. Shared decision making: a model for clinical practice. J Gen Intern Med. 2012;27(10): 1361-1367. doi:10.1007/s11606-012-2077-6
17. Barry MJ, Edgman-Levitan S. Shared decision making-pinnacle of patient-centered care. $N$ Engl J Med. 2012;366(9):780-781. doi:10. 1056/NEJMp1109283

18. Stacey D, Légaré F, Lewis $\mathrm{K}$, et al. Decision aids for people facing health treatment or screening decisions. Cochrane Database Syst Rev. 2017;4(4):CD001431. doi:10.1002/14651858.CD001431.pub5

19. Blaiss MS, Steven GC, Bender B, Bukstein DA, Meltzer EO, Winders T. Shared decision making for the allergist. Ann Allergy Asthma Immunol. 2019;122(5):463-470. doi:10.1016/j.anai.2018.08. 019

20. Allen LA, McIlvennan CK, Thompson JS, et al. Effectiveness of an intervention supporting shared decision making for destination therapy left ventricular assist device: the DECIDE-LVAD randomized clinical trial. JAMA Intern Med. 2018;178(4):520-529. doi:10.1001/ jamainternmed.2017.8713

21. Wilson SR, Strub P, Buist AS, et al. Shared treatment decision making improves adherence and outcomes in poorly controlled asthma. Am J Respir Crit Care Med. 2010;181(6):566-577. doi:10. 1164/rccm.200906-0907OC

22. Lofland JH, Johnson PT, Ingham MP, Rosemas SC, White JC, Ellis L. Shared decision-making for biologic treatment of autoimmune disease: influence on adherence, persistence, satisfaction, and health care costs. Patient Prefer Adherence. 2017;11:947-958. doi:10.2147/PPA.S133222

23. Arterburn D, Wellman R, Westbrook E, et al. Introducing decision aids at Group Health was linked to sharply lower hip and knee surgery rates and costs. Health Aff (Millwood). 2012;31(9):20 94-2104. doi:10.1377/hlthaff.2011.0686

24. Veroff D, Marr A, Wennberg DE. Enhanced support for shared decision making reduced costs of care for patients with preference-sensitive conditions. Health Aff (Millwood). 2013;32(2):2 85-293. doi:10.1377/hlthaff.2011.0941

25. Fiks AG, Mayne SL, Karavite DJ, et al. Parent-reported outcomes of a shared decision-making portal in asthma: a practice-based RCT. Pediatrics. 2015;135(4):e965-973. doi:10.1542/peds.2014-3167

26. Elwyn G, Durand MA, Song J, et al. A three-talk model for shared decision making: multistage consultation process. BMJ. 2017;359: j4891. doi:10.1136/bmj.j4891
Journal of Asthma and Allergy

\section{Publish your work in this journal}

The Journal of Asthma and Allergy is an international, peer-reviewed open-access journal publishing original research, reports, editorials and commentaries on the following topics: Asthma; Pulmonary physiology; Asthma related clinical health; Clinical immunology and the immunological basis of disease; Pharmacological interventions and new therapies. The manuscript management system is completely online and includes a very quick and fair peer-review system, which is all easy to use. Visit http://www.dovepress.com/testimonials.php to read real quotes from published authors. 